\title{
Large-Scale Multi-View Subspace Clustering in Linear Time
}

\author{
Zhao Kang, ${ }^{1}$ Wangtao Zhou, ${ }^{1}$ Zhitong Zhao, ${ }^{1}$ Junming Shao, ${ }^{1}$ Meng Han, ${ }^{2 *}$ Zenglin Xu ${ }^{1,3 *}$ \\ ${ }^{1}$ School of Computer Science and Engineering, University of Electronic Science and Technology of China, China \\ ${ }^{2}$ School of Management and Economics, University of Electronic Science and Technology of China, China \\ ${ }^{3}$ Centre for Artificial Intelligence, Peng Cheng Lab, Shenzhen 518055, China \\ \{zkang, zlxu\}@uestc.edu.cn
}

\begin{abstract}
A plethora of multi-view subspace clustering (MVSC) methods have been proposed over the past few years. Researchers manage to boost clustering accuracy from different points of view. However, many state-of-the-art MVSC algorithms, typically have a quadratic or even cubic complexity, are inefficient and inherently difficult to apply at large scales. In the era of big data, the computational issue becomes critical. To fill this gap, we propose a large-scale MVSC (LMVSC) algorithm with linear order complexity. Inspired by the idea of anchor graph, we first learn a smaller graph for each view. Then, a novel approach is designed to integrate those graphs so that we can implement spectral clustering on a smaller graph. Interestingly, it turns out that our model also applies to single-view scenario. Extensive experiments on various largescale benchmark data sets validate the effectiveness and efficiency of our approach with respect to state-of-the-art clustering methods.
\end{abstract}

\section{Introduction}

During the last decade, subspace clustering has been explored extensively due to its promising performance (Huang et al. 2019; Peng, Kang, and Cheng 2017). Basically, it is based on the assumption that the data points are drawn from multiple low-dimensional subspaces and each group fits into one of the low-dimensional subspaces. Significant progress has been made to uncover such underlying subspaces.

Among the various subspace clustering approaches, spectral clustering based methods such as low-rank representation (LRR) (Liu et al. 2013; Kang, Peng, and Cheng 2015) and sparse subspace clustering (SSC) (Elhamifar and Vidal 2013) have achieved great success. SSC finds a sparse representation of data points while LRR finds the low-rank structure of subspace. Afterwards, the spectral clustering algorithm is performed on such new representation $(\mathrm{Ng}$, Jordan, and Weiss 2002). For $n$ data points, the first step typically takes $\mathcal{O}\left(n^{2}\right)$ or $\mathcal{O}\left(n^{3}\right)$ time while the second step requires $\mathcal{O}\left(n^{3}\right)$ or at least $\mathcal{O}\left(n^{2} k\right)$ time, where $k$ is the number of clusters. Therefore, these two time comsuming steps debar

\footnotetext{
${ }^{*}$ Corresponding author Copyright (C) 2020, Association for the Advancement of Artificial Intelligence (www.aaai.org). All rights reserved.
}

the application of subspace clustering from large-scale data sets.

Recently, much work have been developed to accelerate subspace clustering. For example, (Wang et al. 2014) proposes a data selection approach; a scalable SSC based on orthogonal matching pursuit is developed in (You, Robinson, and Vidal 2016); a fast solver for SSC is proposed in (Pourkamali-Anaraki and Becker 2018); accelerated LRR is also developed (Fan et al. 2018; Xiao et al. 2015); a sampling approach is used in ( $\mathrm{Li}$ and Zhao 2017). Unfortunately, they all target for single-view scenario and can not deal with multi-view data.

With the advance of information technology, many practical data come from multiple channels or appear in muliple modalities, i.e., the same object can be described in different kinds of features (Kang et al. 2019c; Yin et al. 2018; Zhan et al. 2018; Liu et al. 2019; Yao et al. 2019; Tang et al. 2019). Different feature sets often characterize different yet complementary information. For instance, an image can have heterogeneous features, such as Garbor, HoG, SIFT, GIST, and LBP; an artical can be written in different languages. Accordingly, it is crucial to develop multi-view learning method to incorporate the useful informaiton from different views.

There has been growing interest in developing multiview subspace clustering (MVSC) algorithms. For example, MVSC (Gao et al. 2015) method learns a graph representation for each view and all graphs are assumed to share a unique cluster matrix; (Cao et al. 2015) applies a diversity term to explore the complementarity information; (Luo et al. 2018) explores both consistency and specificity of different views; (Wang et al. 2019; Zhang et al. 2017) perform MVSC in latent representation; (Brbić and Kopriva 2018) imposes both low-rank and sparsity constraints on the graph; (Kang et al. 2019a; 2020) learn one partition for each view and them perform a fusion in partition space. In terms of clustering accuracy, these methods are attractive. Nevertheless, their computation efficiency, at least $\mathcal{O}\left(n^{2} k\right)$ complexity, is largely ignored. In the era of big data, it has been witnessed that many applications involve large-scale multi-view data. Therefore, their severely unaffordable complexity precludes MVSC from real-world applications with big data. More- 
over, existing single-view subspace clustering acceleration techniques do not apply to multi-view scenarios owing to the heterogeneous nature of multi-view data.

To fill the gap, we need to address two challenges. First, how to avoid constructing $n \times n$ graph for multi-view data, which requires a lot of time and storage. Second, how to circumvent the computationally expensive step-spectral clustering with $\mathcal{O}\left(n^{2} k\right)$ complexity. To this end, we introduce a novel large-scale multi-view subspace clustering (LMVSC) method, which can greatly reduce the computational complexity as well as improve the clustering performance. First, to address the large-scale graph construction problem, we select a small number of the instances as anchors, and then a smaller graph is built for each view. Second, a novel way to integrate mutiple graphs is designed so that we can implement spectral clustering on a small graph.

The contributions of this paper mainly include:

- We make the first attempt towards large-scale multi-view subspace clustering. Our proposed method can be solved in linear time, while existing methods demand square or cubic time in the number of data instance.

- Extensive experiments in terms of data sets and comparison methods validate the effectiveness and efficiency of the proposed algorithm.

- As a special case, our method also applies to single-view subspace clustering task. It gives promising performance on very large-scale data.

\section{Preliminaries Multi-view Subspace Clustering}

Subspace clustering expresses each data point as a linear combination of other points and minimizes the reconstruction loss to obtain the combination coefficient. This coefficient is treated as the similarity between corresponding points. Mathematically, given data $X \in \mathcal{R}^{d \times n}$ with $d$ features and $n$ samples, subspace clustering solves the following problem (Liu et al. 2013; Elhamifar and Vidal 2013; Kang et al. 2019d):

$$
\min _{Z}\|X-X S\|_{F}^{2}+\alpha f(S) \quad \text { s.t. } \quad S \geq 0, S \mathbf{1}=\mathbf{1},
$$

where $f(\cdot)$ is a certain regularizer function, $\alpha>0$ is a balance parameter, and $\mathbf{1}$ is a vector with all ones. The constraints require that all $S_{i j}$ is nonnegative and $\sum_{j} S_{i j}=1$. $S$ is often called similarity graph matrix. It is obvious that $S$ has size of $n \times n$, which poses a big challenge with scalability to large-scale data sets.

Recently, multi-view subspace clustering (MVSC) has attracted much attention. Basically, for multi-view data $X=$ $\left[X^{1} ; \cdots ; X^{i} ; \cdots ; X^{v}\right] \in \mathcal{R}^{\sum_{i=1}^{v} d_{i} \times n}, \operatorname{MVSC}$ (Gao et al. 2015; Cao et al. 2015; Kang et al. 2019c) solves:

$$
\min _{S^{i}} \sum_{i=1}^{v}\left\|X^{i}-X^{i} S^{i}\right\|_{F}^{2}+\alpha f\left(S^{i}\right) \text { s.t. } S^{i} \geq 0, S^{i} \mathbf{1}=\mathbf{1} .
$$

With different forms of $f$, Eq. (2) gives solutions with different properties. For example, (Wang et al. 2016b) encourages similarity between pairs of graphs; (Cao et al. 2015) emphasizes the complementarity. With these multiple graphs, (Gao et al. 2015) assumes they produce the same clustering; (Cao et al. 2015; Wang et al. 2016b) perform spectral clustering on averaged graph. All these MVSC methods take at least $\mathcal{O}\left(n^{2} k\right)$ time, hence they are generally computational prohibtive.

\section{Anchor Graph}

Anchor graph is an efficient way to construct the large-scale graph matrix (Chen and Cai 2011; Liu, He, and Chang 2010; Wang et al. 2016a; Han, Xiong, and Nie 2017). Specifically, a subset of $m$ points $(m \ll n) A=\left[a_{1}, a_{2}, \cdots, a_{m}\right] \in$ $\mathcal{R}^{d \times m}$ are chosen based on a $k$-means clustering or random sampling on the original data set. Each point plays a role as a landmark which approximates the neighborhood structure. Then, a sparse affinity matrix $Z \in \mathcal{R}^{n \times m}$ is constructed between the landmarks and the original data as follows:

$$
Z_{i j}= \begin{cases}\frac{K_{\delta}\left(x_{i}, a_{j}\right)}{\sum_{j^{\prime} \in<i>} K_{\delta}\left(x_{i}, a_{j^{\prime}}\right)}, & j \in<i> \\ 0, & \text { otherwise }\end{cases}
$$

where $\langle i\rangle$ indicates the indexes of $r(r<m)$ nearest anchors around $x_{i}$. The function $K_{\delta}(\cdot)$ is the commonly used Gaussian kernel with bandwidth parameter $\delta$. Subsequently, the doubly-stochastic similarity matrix $S \in \mathcal{R}^{n \times n}$, the summation of each column and each row equal to one, is built based on:

$$
S=\hat{Z} \hat{Z}^{\top} \text { where } \hat{Z}=Z \Sigma^{-1 / 2},
$$

where $\Sigma$ is a diagonal matrix with the entry $\Sigma_{i i}=$ $\sum_{j=1}^{n} Z_{j i}$.

We can see that the above graph construction approach is extremely efficient since only $\mathcal{O}(m n)$ distances are considered. However, this heuristic strategy has one inherent drawback which is always ignored, i.e., quality of the built graph heavily depends on the exponential function. In general, it might not be appropriate to the structure of the data space (Shakhnarovich 2005; Kang et al. 2019b). Furthermore, it is also challenging to find a good kernel parameter without any supervision information. Consequently, the downstream task might not be able to obtain a good performance.

To obtain a similarity measure tailored to the problem at hand, a principled way is to learn it from data (Kang et al. 2019e). In this paper, inspired by the idea of anchor graph, we use a smaller graph $Z$ to approximate the full $n \times n$ graph matrix. Different from existing approach, we adaptively learn $Z$ from raw data by solving an optimization problem.

\section{Methodology}

For large-scale data, there is much redundancy in the data; a small number of the instances are sufficient for reconstructing the underlying subspaces. Similar to anchor strategy, we use a smaller matrix $Z^{i} \in \mathcal{R}^{n \times m}(m \ll n)$ to approximate the full $n \times n$ matrix $S^{i}$. Specifically, a set of $m$ landmarks $A^{i}=\left\{a_{j}\right\}_{j \in[1, m]}$ (cluster centers) are obtained through $k$ means clustering on the data $X^{i}$. Then, $Z^{i}$ is built between the landmarks $A^{i}$ and $X^{i}$. 
Rather than using the handcrafted similarity measure (3), we learn $Z^{i}$ from data. For multi-view data, we solve the following problem:

$$
\begin{gathered}
\min _{Z^{i}} \sum_{i=1}^{v}\left\|X^{i}-A^{i}\left(Z^{i}\right)^{\top}\right\|_{F}^{2}+\alpha\left\|Z^{i}\right\|_{F}^{2} \\
\text { s.t. } 0 \leq Z^{i},\left(Z^{i}\right)^{\top} \mathbf{1}=\mathbf{1} .
\end{gathered}
$$

Compared to Eq. (2), we only consider the similarities between $m n$ points instead of $n^{2}$, so the complexity is remarkably reduced. Problem (5) can be easily solved via convex quadratic programming.

Given a set of $v$ graphs $\left\{Z^{i}\right\}_{i \in[1, v]}$, our goal is to merge them so as to make use of the rich information contained in $v$ views. Obviously, it is not feasible to run spectral clustering using $\left\{Z^{i}\right\}_{i \in[1, v]}$ since their size is not $n \times n$ anymore. For multi-view case, we can follow a similar procedure as the traditional method, i.e., apply Eq. (4) to restore the $n \times n$ graph $S^{i}$ and then take average,

$$
\bar{S}=\frac{\sum_{i} S^{i}}{v}
$$

In the sequel, spectral clustering is implemented to achieve embedding $Q \in \mathcal{R}^{n \times k}$, i.e.,

$$
\max _{Q} \operatorname{Tr}\left(Q^{\top} \bar{S} Q\right) \quad \text { s.t. } \quad Q^{\top} Q=\mathbf{I} .
$$

Its solution is the $k$ eigenvectors corresponding to the largest $k$ eigenvalues of $\bar{S}$. However, the eigen decomposition takes at least $\mathcal{O}\left(n^{2} k\right)$ time.

In this paper, relying on proposition 1 (Chen and Cai 2011; Affeldt, Labiod, and Nadif 2019), we present an alternative approach to compute the $k$ left singular vectors of the concatenated matrix $\bar{Z} \in \mathcal{R}^{n \times m v}$,

$$
\bar{Z}=\frac{1}{\sqrt{v}}\left[\hat{Z}^{1}, \cdots, \hat{Z}^{i}, \cdots, \hat{Z}^{v}\right] .
$$

Since $m v \ll n$, using $\bar{Z}$ instead of $\bar{S}$ naturally reduces the computational cost of $Q$.

Proposition 1. Given a set of similarity matrices $\left\{S^{i}\right\}_{i \in[1, v]}$, each of them can be decomposed as $Z^{i}\left(Z^{i}\right)^{\top}$. Define $\bar{Z}=\frac{1}{\sqrt{v}}\left[Z^{1}, \cdots, Z^{i}, \cdots, Z^{v}\right] \in \mathcal{R}^{n \times m v}$, its Singular Value Decomposition (SVD) is $U \Lambda V^{\top}$ (with $U^{\top} U=\mathbf{I}$, $\left.V^{\top} V=\mathbf{I}\right)$. We have,

$$
\max _{Q^{\top} Q=\mathbf{I}} \operatorname{Tr}\left(Q^{\top} \bar{S} Q\right) \Leftrightarrow \min _{Q^{\top} Q=\mathbf{I}, H}\left\|\bar{Z}-Q H^{\top}\right\|_{F}^{2} .
$$

And, the optimal solution $Q^{*}$ is equal to $U$.

Proof. Based on the second term of Eq. (9), one can easily show that $H^{*}=\bar{Z}^{\top} Q$. Plugging the value of $H^{*}$ into Eq. (9), the following equivalences hold

$$
\begin{aligned}
\min _{Q^{\top} Q=\mathbf{I}, H}\left\|\bar{Z}-Q H^{\top}\right\|_{F}^{2} & \Leftrightarrow \min _{Q^{\top} Q=\mathbf{I}}\left\|\bar{Z}-Q Q^{\top} \bar{Z}\right\|_{F}^{2} \\
& \Leftrightarrow \max _{Q^{\top} Q=\mathbf{I}} \operatorname{Tr}\left(Q^{\top} \bar{Z} \bar{Z}^{\top} Q\right) \\
& \Leftrightarrow \max _{Q^{\top} Q=\mathbf{I}} \operatorname{Tr}\left(Q^{\top} \bar{S} Q\right) .
\end{aligned}
$$

Moreover,

$$
\begin{aligned}
\bar{S}=\bar{Z} \bar{Z}^{\top} & =\left(U \Lambda V^{\top}\right)\left(U \Lambda V^{\top}\right)^{\top} \\
& =U \Lambda\left(V^{\top} V\right) \Lambda U^{\top} \\
& =U \Lambda^{2} U^{\top} .
\end{aligned}
$$

Thereby the left singular vectors of $\bar{Z}$ are the same as the eigenvectors of $\bar{S}$.

\begin{tabular}{l}
\hline Algorithm 1: LMVSC algorithm \\
\hline Input: Multi-view data matrix $X^{1}, \cdots, X^{v}$, cluster \\
number $k$, parameter $\alpha$, anchors $\left\{A^{i}\right\}_{i \in[1, v]}$ \\
Step 1: Solve problem (5); \\
Step 2: Construct $\bar{Z}$ as (8); \\
Step 3: Compute $Q$ by performing SVD on $\bar{Z}$. \\
Output: Run $k$-means on $Q$ to achieve final clustering. \\
\hline
\end{tabular}

The complete procedures for our LMVSC method is summarized in Algorithm 1.

Claim 1. As a special case, the proposed LMVSC algorithm is also suitable to single-view data.

Proof. It is easy to observe that our proposed precedure also works for single view data, i.e., $v=1$ and $\bar{Z}=\hat{Z}$ in Eq. (8).

\section{Complexity Analysis}

LMVSC benefits from the low complexity of the anchor strategy for the graph construction and the eigen decomposition on a low-dimensional matrix. Specifically, the construction of $Z^{i} s$ takes $\mathcal{O}\left(n m^{3} v\right)$, where $n$ is the number of data points, $m$ is the number of anchors $(m \ll n), v$ is the view number. In experiments, we apply the built-in matlab function quadprog to solve (5), in which inter-point method with complexity $\mathcal{O}\left(\mathrm{m}^{3}\right)$ is used (Ye and Tse 1989). It is worth mentioning that the construction of $Z^{i} s$ can be easily parallelized over multiple cores, leading to more efficient computation. The computational cost for the $Q$ embedding induces a computational complexity of $\mathcal{O}\left(m^{3} v^{3}+2 m v n\right)$. In addition, we need additional $\mathcal{O}(n m t p)$ for the $k$-means at the begining for anchor selection and $\mathcal{O}\left(n k^{2} t\right)$ for the last $k$-means on $Q$, where $t$ is the number of iterations, $k$ is the number of clusters, $p=\sum_{i=1}^{v} d_{i}$ is the summation of feature dimensions. Accordingly, our proposed LMVSC method costs time only linear in $n$.

\section{Experiment on Multi-View Data}

In this section, we conduct extensive experiments to assess the performance of the proposed method on real-world data sets.

\section{Data Sets}

We perform experiments on several benchmark data sets: Handwritten, Caltech-101, Reuters, NUS-WIDE-Object. Specifically, Handwritten consists of handwritten digits of 0 
Table 1: Description of the data sets. The dimension of features is shown in parenthesis.

\begin{tabular}{lllll}
\hline View & Handwritten & Caltech7/20 & Reuters & NUS \\
\hline 1 & Profile correlations (216) & Gabor(48) & English (21531) & Color Histogram (65) \\
2 & Fourier coefficients (76) & Wavelet moments (40) & French (24892) & Color moments (226) \\
3 & Karhunen coefficients (64) & CENTRIST (254) & German (34251) & Color correlation (145) \\
4 & Morphological (6) & HOG (1984) & Italian (15506) & Edge distribution (74) \\
5 & Pixel averages (240) & GIST (512) & Spanish (11547) & Wavelet texture (129) \\
6 & Zernike moments (47) & LBP (928) & - & - \\
\hline Data points & 2000 & $1474 / 2386$ & 18758 & 30000 \\
Class number & 10 & $7 / 20$ & 6 & 31 \\
\hline
\end{tabular}

Table 2: Clustering performance on Caltech7 data. "-" denotes some unreasonable values that are close to zero.

\begin{tabular}{|c|c|c|c|c|}
\hline Method & Acc & NMI & Purity & Time (s) \\
\hline E3SC(1) & 0.3060 & 0.1993 & 0.3677 & 10.32 \\
E3SC(2) & 0.3256 & 0.2648 & 0.3582 & 9.84 \\
E3SC(3) & 0.4261 & 0.2534 & 0.4573 & 18.19 \\
E3SC(4) & 0.5509 & 0.4064 & 0.5787 & 82.43 \\
E3SC(5) & 0.5122 & 0.3863 & 0.5598 & 27.80 \\
E3SC(6) & 0.5244 & 0.4273 & 0.5773 & 42.86 \\
SSCOMP(1) & 0.2619 & 0.0640 & 0.2958 & 0.84 \\
SSCOMP(2) & 0.3759 & 0.2017 & 0.4023 & 0.82 \\
SSCOMP(3) & 0.3256 & 0.1354 & 0.3460 & 1.50 \\
SSCOMP(4) & 0.5604 & 0.4631 & 0.6357 & 5.35 \\
SSCOMP(5) & 0.4715 & - & - & 2.11 \\
SSCOMP(6) & 0.5000 & 0.3688 & 0.5577 & 3.00 \\
AMGL & 0.4518 & 0.4243 & 0.4674 & 20.12 \\
MLRSSC & 0.3731 & 0.2111 & 0.4145 & 22.26 \\
MSC_IAS & 0.3976 & 0.2455 & 0.4444 & 57.18 \\
LMVSC & $\mathbf{0 . 7 2 6 6}$ & $\mathbf{0 . 5 1 9 3}$ & $\mathbf{0 . 7 5 1 7}$ & 135.79 \\
\hline
\end{tabular}

to 9 from UCI machine learning repository. Caltech-101 is a data set of images for object recognition. Following previous work, two subsets, caltech7 and caltech20, are used. Reuters contains documets written in five different languages and their translations. A subset written in English and all their translations are used here. NUS-WIDE-Object (NUS) is also a object recognition database. More detailed information about these data is shown in Table 1.

\section{Clustering Evaluation}

We compare $\mathrm{LMVSC}^{1}$ with several recent work as stated bellow:

Efficient Solvers for Sparse Subspace Clustering (E3SC): recent subspace clustering method proposed in (Pourkamali-Anaraki and Becker 2018). We run E3SC on each view and report its performance $\mathrm{E} 3 \mathrm{SC}(\cdot)$.

Scalable Sparse Subspace Clustering by Orthogonal Matching Pursuit (SSCOMP): one of the representative large-scale subspace clustering method developed in (You, Robinson, and Vidal 2016). We run it on each single view.

Parameter-Free Auto-Weighted Multiple Graph Learning (AMGL): one of the state-of-the-art multi-view spectral clustering method proposed in (Nie et al. 2016).

Multi-view Low-rank Sparse Subspace Clustering

\footnotetext{
${ }^{1}$ Code is available at https://github.com/sckangz/LMVSC
}

Table 3: Clustering performance on Caltech20 data.

\begin{tabular}{|c|c|c|c|c|}
\hline Method & Acc & NMI & Purity & Time (s) \\
\hline E3SC(1) & 0.2531 & 0.2670 & 0.2657 & 27.71 \\
E3SC(2) & 0.2670 & 0.3248 & 0.2993 & 26.81 \\
E3SC(3) & 0.2921 & 0.3358 & 0.3282 & 46.69 \\
E3SC(4) & 0.4107 & $\mathbf{0 . 5 5 7 6}$ & 0.4908 & 180.70 \\
E3SC(5) & 0.4162 & 0.4834 & 0.4644 & 61.32 \\
E3SC(6) & 0.4845 & 0.4976 & 0.5444 & 99.62 \\
SSCOMP(1) & 0.1928 & 0.1359 & 0.2087 & 1.66 \\
SSCOMP(2) & 0.2511 & 0.2338 & 0.2955 & 1.56 \\
SSCOMP(3) & 0.1945 & 0.1319 & 0.2360 & 2.75 \\
SSCOMP(4) & 0.3667 & 0.4487 & 0.4288 & 10.02 \\
SSCOMP(5) & 0.2930 & 0.1681 & $\mathbf{0 . 6 0 1 0}$ & 4.45 \\
SSCOMP(6) & 0.3567 & 0.3735 & 0.4346 & 5.88 \\
AMGL & 0.3013 & 0.4054 & 0.3164 & 77.63 \\
MLRSSC & 0.2821 & 0.2670 & 0.3039 & 607.28 \\
MSC_IAS & 0.3127 & 0.3138 & 0.3374 & 93.87 \\
LMVSC & $\mathbf{0 . 5 3 0 6}$ & 0.5271 & 0.5847 & 342.97 \\
\hline
\end{tabular}

(MLRSSC): recent method (Brbić and Kopriva 2018) which outperforms many multi-view spectral clustering methods, e.g., (Xia et al. 2014).

Multi-view Subspace Clustering with Intactness-aware Similarity (MSC_IAS): another recent multi-view subspace clustering method proposed in (Wang et al. 2019), which reports improvements against a number of MVSC methods, e.g., (Zhang et al. 2017).

All our experiments are implemented on a computer with a 2.6GHz Intel Xeon CPU and 64GB RAM, Matlab R2016a. For fair comparison, we follow the experimental setting described in respective papers and tune the parameters to obtain the best performance. We search anchor number $m$ in the range $[k, 50,100]$. The motivation behind this is that the number of points required for revealing the underlying subspaces should not be less than the number of subspaces, i.e., the cluster number $k$. In addition, some other factors, such as subspace's intrinsic dimensions, noise level could also influence the anchor number. We select $\alpha$ from the range $[0.001,0.01,0.1,1,10]$. We assess the performance with accuracy (Acc), normalized mutual information (NMI), purity, and running time.

\section{Experimental Results}

Tables 2-6 show the clustering results of all methods on five data sets. Due to out-of-memory issue, many comparison methods, e.g., E3SC and MLRSSC, are not appliable 
Table 4: Clustering performance on Handwritten data.

\begin{tabular}{|c|c|c|c|c|}
\hline Method & Acc & NMI & Purity & Time (s) \\
\hline E3SC(1) & 0.7555 & 0.7491 & 0.8385 & 29.94 \\
E3SC(2) & 0.5790 & 0.5279 & 0.6200 & 20.80 \\
E3SC(3) & 0.7300 & 0.7226 & 0.8095 & 19.47 \\
E3SC(4) & 0.4530 & 0.4623 & 0.5290 & 16.07 \\
E3SC(5) & 0.7555 & 0.7153 & 0.8345 & 31.52 \\
E3SC(6) & 0.6345 & 0.6189 & 0.6825 & 18.69 \\
SSCOMP(1) & 0.4680 & 0.3939 & 0.5485 & 2.21 \\
SSCOMP(2) & 0.3640 & 0.2913 & 0.4475 & 2.07 \\
SSCOMP(3) & 0.2430 & 0.1536 & 0.4475 & 2.39 \\
SSCOMP(4) & 0.3005 & 0.3191 & 0.3250 & 0.81 \\
SSCOMP(5) & 0.5370 & 0.4911 & 0.6640 & 2.23 \\
SSCOMP(6) & 0.3115 & 0.1586 & 0.3670 & 1.37 \\
AMGL & 0.8460 & $\mathbf{0 . 8 7 3 2}$ & 0.8710 & 67.58 \\
MLRSSC & 0.7890 & 0.7422 & 0.8375 & 52.44 \\
MSC_IAS & 0.7975 & 0.7732 & 0.8755 & 80.78 \\
LMVSC & $\mathbf{0 . 9 1 6 5}$ & 0.8443 & $\mathbf{0 . 9 1 6 5}$ & 10.55 \\
\hline
\end{tabular}

Table 5: Clustering performance on Reuters data.

\begin{tabular}{|c|c|c|c|c|}
\hline Method & Acc & NMI & Purity & Time (s) \\
\hline SSCOMP(1) & 0.2714 & - & - & 4721.90 \\
SSCOMP(2) & 0.2730 & - & - & 5070.80 \\
SSCOMP(3) & 0.2735 & - & - & 4524.10 \\
SSCOMP(4) & 0.2719 & - & - & 4515.70 \\
SSCOMP(5) & 0.3128 & - & - & 4162.40 \\
AMGL & 0.1672 & - & - & 32397 \\
MSC_IAS & 0.5063 & 0.2759 & 0.6031 & 7015.7 \\
LMVSC & $\mathbf{0 . 5 8 9 0}$ & $\mathbf{0 . 3 3 4 6}$ & $\mathbf{0 . 6 1 4 5}$ & 130.73 \\
\hline
\end{tabular}

to Reuters and NUS data sets which are more than 10,000 samples. Therefore, they are not listed in Table 5 and Table 6 . This demonstrates that the proposed method is low space complexity. In terms of accuracy, our method constantly outperforms others and the average improvement is at least $7 \%$ over the second highest value. For NMI and purity, our method achieves comparable or even better performance than the other methods. This demonstrates the consistency and stability of our method. Taken Caltech7 and Handwritten as examples, we visualize their clustering results based on t-SNE technique in Figure 1. We can clearly observe the cluster pattern.

For running time comparison, our method can finish all data sets in several minutes. Our method is up to several orders of magnitude faster than other multi-view methods on large data sets. For example, MSC_IAS consumes almost 13

Table 6: Clustering performance on NUS data.

\begin{tabular}{|c|c|c|c|c|}
\hline Method & Acc & NMI & Purity & Time (s) \\
\hline SSCOMP(1) & 0.1153 & 0.0755 & 0.1412 & 53.63 \\
SSCOMP(2) & 0.1206 & - & - & 102.64 \\
SSCOMP(3) & 0.1138 & - & - & 90.88 \\
SSCOMP(4) & 0.0950 & - & - & 64.26 \\
SSCOMP(5) & 0.0756 & - & - & 83.83 \\
MSCIAS & 0.1548 & $\mathbf{0 . 1 5 2 1}$ & 0.1675 & 45386 \\
LMVSC & $\mathbf{0 . 1 5 5 3}$ & 0.1295 & $\mathbf{0 . 1 9 8 2}$ & 165.39 \\
\hline
\end{tabular}

hours to finish NUS data, while our method only takes 3 minutes. Note that the state-of-the-art scalable method SSCOMP takes extremely long time to run reuters data, which is due to the data's high dimensionality. According to our observation, the fluctuation of our execution time is mainly caused by the number of anchors. For instance, Handwritten achieves the best results when the fewest anchors are used, i.e., cluster number 10. Consequently, it takes the shortest time.

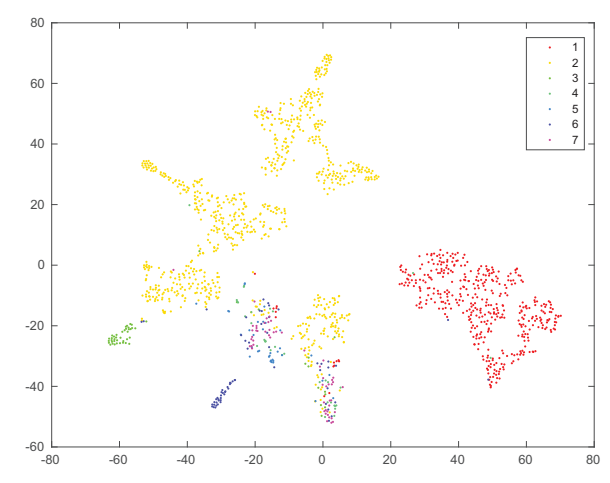

(a) Caltech7

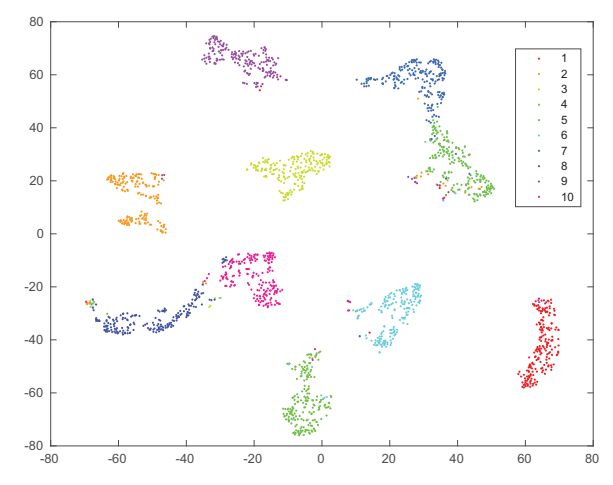

(b) Handwritten

Figure 1: Visualization of some clustering results.

\section{Robustness Study}

To examine the robustness of our method, we perform additional experiments by adding three commonly seen kinds of noise. We choose the famous MNIST database, which consists of gray-scale images of size $28 \times 28$. There are 70000 samples in total. Since it is a single-view data, we construct a multi-view data set by adding different levels of noise to different views. Specifically, we first corrupt MNIST data by adding Gaussian noise with variance $0.01,0.03$, and 0.05 ; Salt\&Pepper noise with density $0.05,0.1$, and 0.2 ; Speckle noise with variance $0.05,0.1$, and 0.15 , respectively. Then, we concatenate them to form three three-view data sets. Some example images are shown in Figures 2-4. For these large data sets, we find that only SSCOMP and our method 
Table 7: Clustering performance on MNIST data.

\begin{tabular}{|c|c|c|c|c|c|}
\hline Noise & Method & Acc & NMI & Purity & Time (s) \\
\hline \multirow{5}{*}{ Gaussian } & SSCOMP(1) & 0.4300 & 0.4726 & 0.5965 & 1120.80 \\
\cline { 2 - 6 } & SSCOMP(2) & 0.4465 & 0.4749 & 0.5908 & 1151.50 \\
\cline { 2 - 6 } & SSCOMP(3) & 0.4418 & 0.4754 & 0.5920 & 1130.30 \\
\cline { 2 - 6 } Speckle & LMVSC & $\mathbf{0 . 5 5 6 5}$ & $\mathbf{0 . 5 0 9 6}$ & $\mathbf{0 . 6 2 8 2}$ & 55.17 \\
\hline \multirow{5}{*}{ Salt\&Pepper } & SSCOMP(1) & 0.4417 & 0.4770 & 0.5967 & 1314.90 \\
\cline { 2 - 6 } & SSCOMP(2) & 0.4560 & 0.4795 & 0.5995 & 1287.70 \\
\cline { 2 - 6 } & SSCOMP(3) & 0.4556 & 0.4855 & 0.6043 & 1303.40 \\
\cline { 2 - 6 } & LMVSC & $\mathbf{0 . 5 9 2 0}$ & $\mathbf{0 . 5 1 7 8}$ & $\mathbf{0 . 6 1 8 3}$ & 66.01 \\
\hline & SSCOMP(1) & 0.4536 & 0.4782 & 0.5964 & 1181.90 \\
\cline { 2 - 6 } & SSCOMP(2) & 0.4513 & 0.4822 & 0.6021 & 1306.10 \\
\cline { 2 - 6 } & SSCOMP(3) & 0.4816 & 0.4923 & 0.6307 & 1302.00 \\
\cline { 2 - 6 } & LMVSC & $\mathbf{0 . 5 8 8 9}$ & $\mathbf{0 . 5 3 7 4}$ & $\mathbf{0 . 6 5 9 8}$ & 73.33 \\
\hline
\end{tabular}

can handle it, while other recent multi-view clustering methods fail.

According to the results in Table 7, our method obtains better performance than SSCOMP in all metrics. This also verifies the advantage of multi-view learning which can exploit the complementarity of multi-view data. In terms of computation time, our method is 20 times faster than SSCOMP.

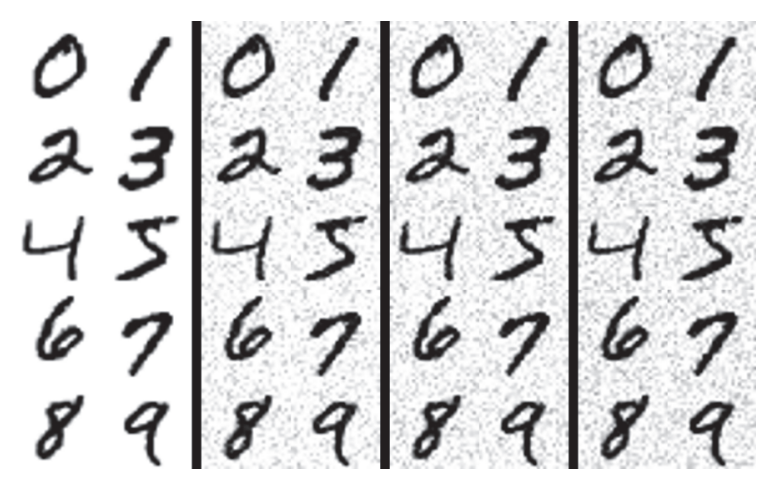

Figure 2: Some sample images of MNIST are shown in the 1st column. Corresponding noisy images contaminated by Gaussian noise with variance $0.01,0.03$, and 0.05 , are displayed in the 2nd, 3rd, and 4th column, respectively.

\section{Parameter Analysis}

During the experiments, we tune two parameters based on grid search, i.e., the number of anchors and $\alpha$. Taking Handwritten and MNIST data sets as examples, we show our model's sensitivity to their values in Figures 5 and 6. We can see that $\alpha$ tends to have a small value since the performance is degraded when $\alpha$ increases. We have similar observation for anchor number. Too many anchors will make themselves less representative and introduce extra errors. Consequently, the performance will be deteriorated.

\section{Experiment on Single-View Data}

We use two large-scale single-view data sets to demonstrate the performance of our algorithm on single-view

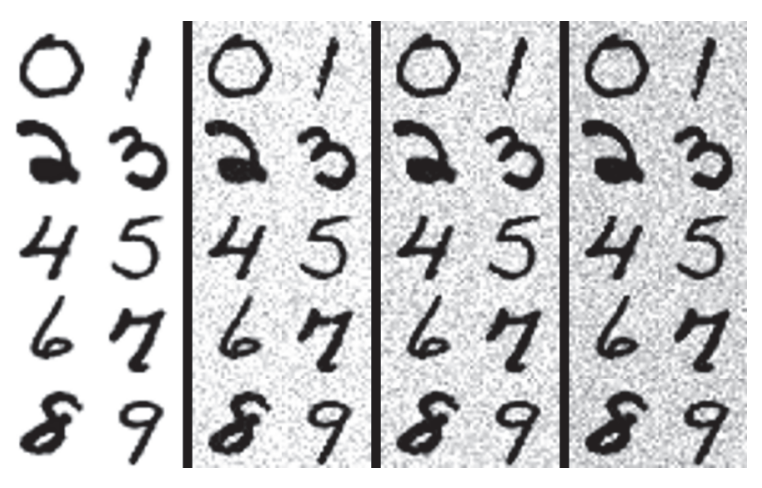

Figure 3: Some sample images of MNIST are shown in the 1 st column. Corresponding noisy images contaminated by Speckle noise with variance $0.05,0.1$, and 0.15 , are displayed in the $2 \mathrm{nd}$, 3rd, and 4th column, respectively.

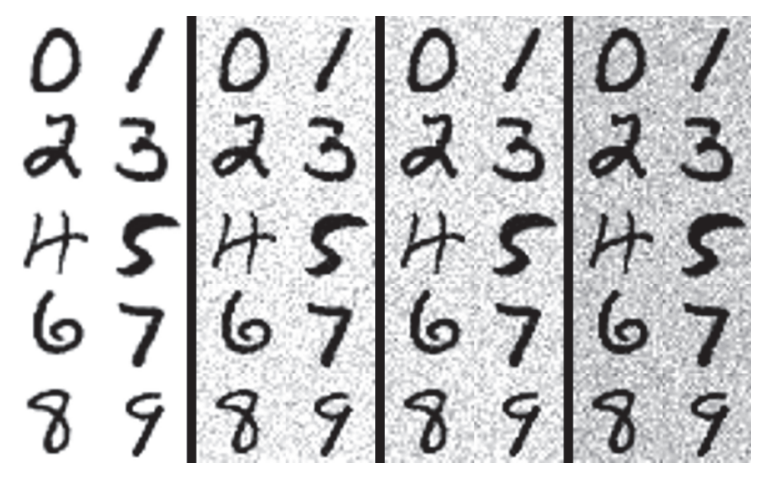

Figure 4: Some sample images of MNIST are shown in the 1 st column. Corresponding noisy images contaminated by Salt\&Pepper noise with density $0.05,0.1$, and 0.2 , are displayed in the $2 \mathrm{nd}$, 3rd, and 4th column, respectively.

Table 8: Description of single-view data sets.

\begin{tabular}{l|cll}
\hline Data Sets & \#Feature & \#Classes & \#Sample \\
\hline RCV1 & 1979 & 103 & 193844 \\
CoverType & 54 & 7 & 581012 \\
\hline
\end{tabular}

Table 9: Results of single-view data sets.

\begin{tabular}{|c|l|c|l|l|l|}
\hline Data Sets & Method & Acc & NMI & Purity & Time (s) \\
\hline \multirow{2}{*}{ RCV1 } & KM & 0.1846 & 0.2447 & 0.2550 & 1569.07 \\
\cline { 2 - 6 } & LMVSC & 0.1929 & 0.2600 & 0.2985 & 1035 \\
\hline \multirow{2}{*}{ CoverType } & KM & 0.2505 & 0.0617 & 0.3039 & 156.72 \\
\cline { 2 - 6 } & LMVSC & 0.3862 & 0.1273 & 0.4889 & 2755.8 \\
\hline
\end{tabular}



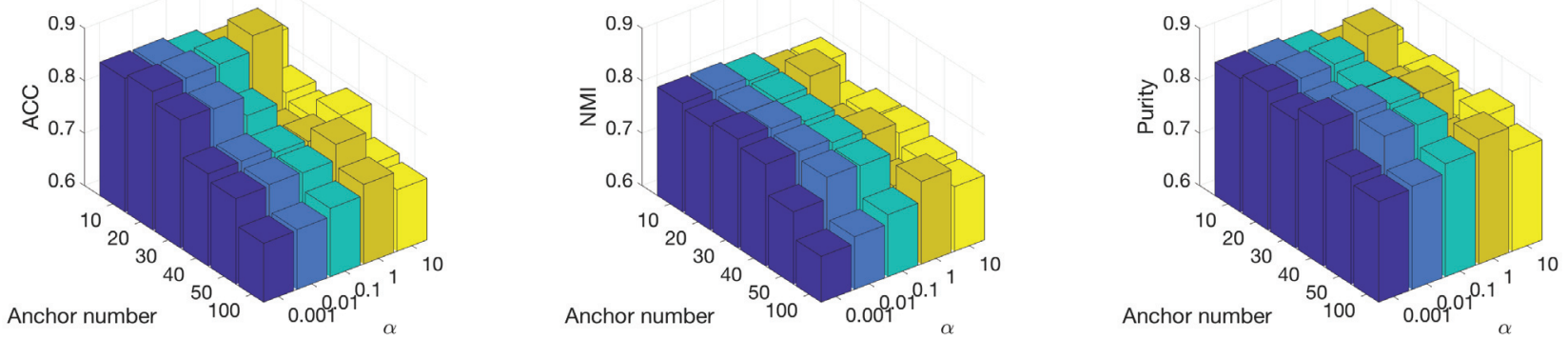

Figure 5: Sensitivity analysis of parameters for our method over Handwritten data set.
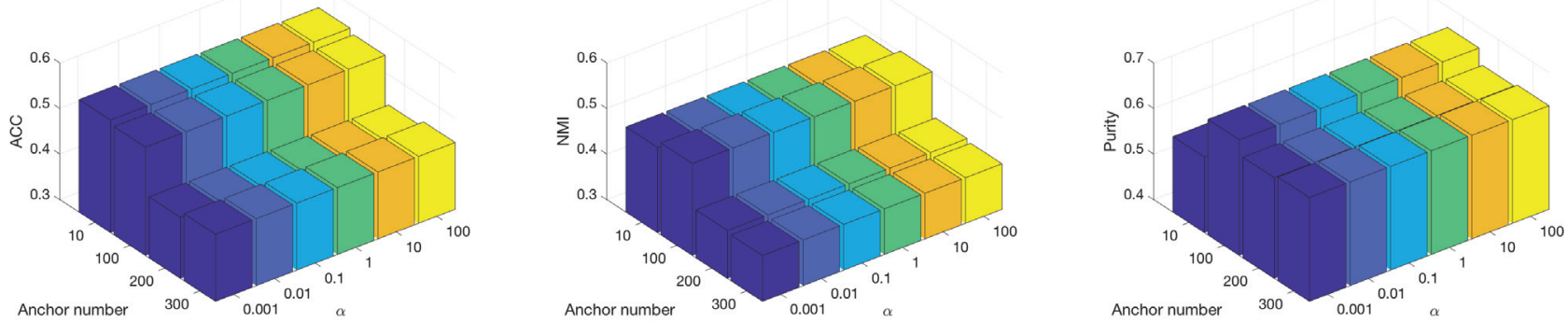

Figure 6: Sensitivity analysis of parameters for our method over MNIST data set.

data. Specifically, RCV1 consists of newswire stories from Reuters Ltd. CovType contains instances for predicting forest cover type from cartographic variables. Their statistics are summarized in Table 8 . We found that SSCOMP can not finish within 24 hours, while many other large-scale subspace clustering (Fan et al. 2018; Xiao et al. 2015) methods run out of memory. For comparison, we include $k$-means $(\mathrm{KM})$ as baseline. From Table 9, we can see that our method improves the performance significantly. Considering the size of data, our computation time is still reasonable. For CoverType, KM runs fast since the cluster number is quite small.

\section{Conclusion}

In this paper, we propose a novel multi-view subspace clustering algorithm. To the best of our knowledge, LMVSC is the very first effort of addressing the large-scale problem of multi-view subspace clustering. Our proposed method has a linear computation complexity, while maintaining high level of clustering accuracy. Specifically, for each view, one smaller graph is built between the raw data points and the generated anchors. Then, a novel integration mechanism is designed to merge those graphs, so that the eigen decomposition can be accelerated significantly. Furthermore, our proposed method also applies to single-view data. Extensive experiments on real data sets verify the effectiveness, efficiency, and robustness of the proposed method against other state-of-the-art techniques.

\section{ACKNOWLEDGMENT}

This paper was in part supported by Grants from the Natural Science Foundation of China (Nos. 61806045 and 61572111) and Fundamental Research Fund for the Central Universities of China (No. ZYGX2017KYQD177).

\section{References}

Affeldt, S.; Labiod, L.; and Nadif, M. 2019. Spectral clustering via ensemble deep autoencoder learning (sc-edae). arXiv preprint arXiv:1901.02291.

Brbić, M., and Kopriva, I. 2018. Multi-view low-rank sparse subspace clustering. Pattern Recognition 73:247-258.

Cao, X.; Zhang, C.; Fu, H.; Liu, S.; and Zhang, H. 2015. Diversity-induced multi-view subspace clustering. In $C V P R$, 586-594.

Chen, X., and Cai, D. 2011. Large scale spectral clustering with landmark-based representation. In AAAI.

Elhamifar, E., and Vidal, R. 2013. Sparse subspace clustering: Algorithm, theory, and applications. IEEE transactions on pattern analysis and machine intelligence 35(11):27652781.

Fan, J.; Tian, Z.; Zhao, M.; and Chow, T. W. 2018. Accelerated low-rank representation for subspace clustering and semi-supervised classification on large-scale data. Neural Networks 100:39-48.

Gao, H.; Nie, F.; Li, X.; and Huang, H. 2015. Multi-view subspace clustering. In ICCV, 4238-4246. 
Han, J.; Xiong, K.; and Nie, F. 2017. Orthogonal and nonnegative graph reconstruction for large scale clustering. In IJCAI, 1809-1815.

Huang, W.; Yin, M.; Li, J.; and Xie, S. 2019. Deep clustering via weighted k-subspace network. IEEE Signal Processing Letters 26:1628-1632.

Kang, Z.; Guo, Z.; Huang, S.; Wang, S.; Chen, W.; Su, Y.; and $\mathrm{Xu}, \mathrm{Z}$. 2019a. Multiple partitions aligned clustering. In IJCAI, 2701-2707.

Kang, Z.; Pan, H.; Hoi, S. C.; and Xu, Z. 2019b. Robust graph learning from noisy data. IEEE Transactions on $C y$ bernetics $1-11$.

Kang, Z.; Shi, G.; Huang, S.; Chen, W.; Pu, X.; Zhou, J. T.; and $\mathrm{Xu}, \mathrm{Z}$. 2019c. Multi-graph fusion for multi-view spectral clustering. Knowledge-Based Systems.

Kang, Z.; Wen, L.; Chen, W.; and Xu, Z. 2019d. Lowrank kernel learning for graph-based clustering. KnowledgeBased Systems 163:510-517.

Kang, Z.; Xu, H.; Wang, B.; Zhu, H.; and Xu, Z. 2019 e. Clustering with similarity preserving. Neurocomputing 365:211-218.

Kang, Z.; Zhao, X.; Shi; Peng, c.; Zhu, H.; Zhou, J. T.; Peng, X.; Chen, W.; and Xu, Z. 2020. Partition level multiview subspace clustering. Neural Networks 122:279-288.

Kang, Z.; Peng, C.; and Cheng, Q. 2015. Robust subspace clustering via smoothed rank approximation. IEEE Signal Processing Letters 22(11):2088-2092.

Li, J., and Zhao, H. 2017. Large-scale subspace clustering by fast regression coding. In IJCAI.

Liu, G.; Lin, Z.; Yan, S.; Sun, J.; Yu, Y.; and Ma, Y. 2013. Robust recovery of subspace structures by low-rank representation. IEEE transactions on pattern analysis and machine intelligence 35(1):171-184.

Liu, X.; Zhu, X.; Li, M.; Wang, L.; Zhu, E.; Liu, T.; Kloft, M.; Shen, D.; Yin, J.; and Gao, W. 2019. Multiple kernel k-means with incomplete kernels. IEEE transactions on pattern analysis and machine intelligence.

Liu, W.; He, J.; and Chang, S.-F. 2010. Large graph construction for scalable semi-supervised learning. In ICML, 679-686.

Luo, S.; Zhang, C.; Zhang, W.; and Cao, X. 2018. Consistent and specific multi-view subspace clustering. In $A A A I$.

Ng, A. Y.; Jordan, M. I.; and Weiss, Y. 2002. On spectral clustering: Analysis and an algorithm. In NIPS, 849-856.

Nie, F.; Li, J.; Li, X.; et al. 2016. Parameter-free autoweighted multiple graph learning: A framework for multiview clustering and semi-supervised classification. In IJCAI, 1881-1887.

Peng, C.; Kang, Z.; and Cheng, Q. 2017. Subspace clustering via variance regularized ridge regression. In $C V P R$, 2931-2940.

Pourkamali-Anaraki, F., and Becker, S. 2018. Efficient solvers for sparse subspace clustering. arXiv preprint arXiv:1804.06291.
Shakhnarovich, G. 2005. Learning task-specific similarity. Ph.D. Dissertation, Massachusetts Institute of Technology.

Tang, C.; Zhu, X.; Liu, X.; and Wang, L. 2019. Cross-view local structure preserved diversity and consensus learning for multi-view unsupervised feature selection. In Proceedings of the AAAI Conference on Artificial Intelligence, volume 33, 5101-5108.

Wang, S.; Tu, B.; Xu, C.; and Zhang, Z. 2014. Exact subspace clustering in linear time. In $A A A I$.

Wang, M.; Fu, W.; Hao, S.; Tao, D.; and Wu, X. 2016a. Scalable semi-supervised learning by efficient anchor graph regularization. IEEE Transactions on Knowledge and Data Engineering 28(7):1864-1877.

Wang, Y.; Zhang, W.; Wu, L.; Lin, X.; Fang, M.; and Pan, S. 2016b. Iterative views agreement: an iterative low-rank based structured optimization method to multi-view spectral clustering. In IJCAI, 2153-2159. AAAI Press.

Wang, X.; Lei, Z.; Guo, X.; Zhang, C.; Shi, H.; and Li, S. Z. 2019. Multi-view subspace clustering with intactness-aware similarity. Pattern Recognition 88:50-63.

Xia, R.; Pan, Y.; Du, L.; and Yin, J. 2014. Robust multi-view spectral clustering via low-rank and sparse decomposition. In $A A A I$.

Xiao, S.; Li, W.; Xu, D.; and Tao, D. 2015. Falrr: A fast low rank representation solver. In CVPR, 4612-4620.

Yao, S.; Yu, G.; Wang, J.; Domeniconi, C.; and Zhang, X. 2019. Multi-view multiple clustering. In IJCAI.

Ye, Y., and Tse, E. 1989. An extension of karmarkar's projective algorithm for convex quadratic programming. Mathematical programming 44(1-3):157-179.

Yin, M.; Gao, J.; Xie, S.; and Guo, Y. 2018. Multiview subspace clustering via tensorial t-product representation. IEEE Transactions on Neural Networks and Learning Systems 30(3):851-864.

You, C.; Robinson, D.; and Vidal, R. 2016. Scalable sparse subspace clustering by orthogonal matching pursuit. In CVPR, 3918-3927.

Zhan, K.; Niu, C.; Chen, C.; Nie, F.; Zhang, C.; and Yang, Y. 2018. Graph structure fusion for multiview clustering. IEEE Transactions on Knowledge and Data Engineering.

Zhang, C.; Hu, Q.; Fu, H.; Zhu, P.; and Cao, X. 2017. Latent multi-view subspace clustering. In CVPR, 4279-4287. 